\title{
Mixed Receptive-Expressive Language Disorder
}

National Cancer Institute

\section{Source}

National Cancer Institute. Mixed Receptive-Expressive Language Disorder. NCI

Thesaurus. Code C92563.

A disorder characterized by an impairment in the development of an individual's expressive and receptive language capabilities which is in contrast to his/her nonverbal intellect. The impairment may be acquired (i.e., due to a brain lesion or head trauma) or developmental (i.e., no known neurological insult). 